\title{
Thermal and Optical Characterization of MicroLED Probes for in vivo Optogenetic Neural Stimulation
}

\author{
Niall McAlinden ${ }^{1 a *}$, David Massoubre ${ }^{1 a *}$, Elliot Richardson ${ }^{1}$, Erdan $_{\text {Gu}}{ }^{1}$, Shuzo Sakata ${ }^{2}$, \\ Martin D. Dawson ${ }^{1}$ and Keith Mathieson ${ }^{1}$ \\ ${ }^{1}$ Institute of Photonics, SUPA, University of Strathclyde, Glasgow, UK \\ 2 Strathclyde Institute of Pharmacy and Biomedical Sciences, University of Strathclyde, Glasgow, UK \\ a Niall McAlinden and David Massoubre should be considered joint first authors \\ *Corresponding Author: niall.mcalinden@strath.ac.uk
}

Compiled February 6, 2013

\begin{abstract}
Within optogenetics there is a need for new compact light sources that are capable of delivering light with excellent spatial, temporal and spectral resolution to deep brain structures. Here, we demonstrate a custom GaN-based LED probe for such applications and the electrical, optical and thermal properties are analyzed. The output power density and emission spectrum were found to be suitable for stimulating channelrhodopsin-2, one of the most common light-sensitive proteins currently used in optogenetics. The LED device produced high light intensities, far in excess of those required to stimulate the light-sensitive proteins within the neurons. Thermal performance was also investigated, illustrating that a broad range of operating regimes in pulsed mode are accessible while keeping a minimum increase in temperature for the brain $\left(0.5^{\circ} \mathrm{C}\right)$. This type of custom device represents a significant step forward for the optogenetics community, allowing multiple bright excitation sites along the length of a minimally-invasive neural probe.

(C) 2013 Optical Society of America

OCIS codes: $170.0170,130.3120,170.2945,230.3670$.
\end{abstract}

Optogenetics is a technique that uses light sensitive proteins to control neural activity [1]. The most commonly used protein is channelrhodopsin-2 (ChR2), which is activated by light at $460 \mathrm{~nm}$ and initiates action potentials in mammalian neurons with ms temporal resolution. However, a key problem limiting the development of this field is getting multiple compact light sources at high spatial, temporal and spectral resolution to deep brain regions. The need for multiple optical sources stems from the desire to probe neighboring neurons, neural circuits and cell types. Neurons are often arranged in laminar structures within morphologically distinct regions of the brain, such as the neocortex. A neural probe with multiple optical sites would be able to sequentially activate these layers while recording probes monitor the resultant neural activity. This would provide a valuable tool to further the understanding of how neural function maps to behavior. Most in vivo studies use a single tapered optical fiber to couple light into these regions [2]. Electrodes can be routinely inserted with the fiber to record the resultant electrical activity $[3,4]$. This method, while certainly providing adequate light intensity, only gives a single target region. If stimulation in several sites is required, several fibers must be inserted, greatly increasing the surgical complexity and the damage to delicate neural tissue. Further to this problem, each fiber requires its own light source which must remain connected to the fiber during the course of the experiment, meaning the subject is not free to move about unhindered. Several different solutions to address these problems have been suggested including; fiber bundles [3], wave guide devices $[5,6]$, LED coupled to a waveguide devices [4] and $\mathrm{mm}$ sized LEDs within a glass pipette [7].

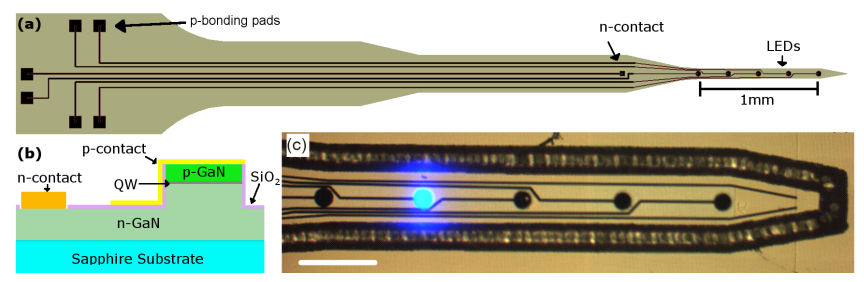

Fig. 1. (a) Layout of LED probe, showing the single ncontact and bonding pads linked with tracks to the pcontacts for each LED. (b) Schematic cross section of the fabricated devices, showing the quantum well (QW) LED structure. (c) Tip of the 5 site LED probe, before final thinning, with a single LED switched on, emission is through the sapphire substrate. Scale bar is $200 \mu \mathrm{m}$.

In this paper a new approach is introduced, a probe with integrated micron-sized LEDs. The main reasons that these LED probe devices have not been pursued before is due to the significant technical challenges involved and a commonly held belief that heat dissipation could damage local brain tissue. Here we examine the optical and thermal properties of LED probe devices, measured under the pulsed regimes required for optogenetic studies and show that these devices provide sufficient power, with excellent spatial, spectral and temporal resolution, while not increasing the temperature of local brain tissue by more than $0.5^{\circ} \mathrm{C}$. Since the optical sites are integrated onto the probe and electrically activated, the device could potentially be extended to allow a freely moving subject through wireless communication. The in- 
tegration of more LEDs onto the probe is only limited by the width of the metallic tracks, which can be as narrow as a few hundred nanometers. This gives the possibility of having 10s of LEDs on a probe without having a probe width that will damage the neural tissue.

The LED probe was fabricated from a commercial GaN $450 \mathrm{~nm}$ LED wafer (SuperNovaOpto). The probe is designed to have a total length of $7 \mathrm{~mm}$ (fig. 1(a)). The tip is $1.3 \mathrm{~mm}$-long and $80 \mu \mathrm{m}$-wide and was designed to minimize tissue damage during insertion (fig. 1(c)). Each tip contains 5 LEDs with a pitch of $250 \mu \mathrm{m}$ and a diameter of $40 \mu \mathrm{m}$. The other end of the probe contains bonding pads allowing each LED to be individually addressed. The following process flow was used to manufacture the probes as adapted from Zhang et al. [8]. After a de-oxidation step, $30 \mathrm{~nm}$ of palladium was deposited by electron beam evaporation, followed by a rapid thermal annealing at $300^{\circ} \mathrm{C}$ for $120 \mathrm{~s}$ in a pure nitrogen ambient. This forms a semi-transparent ohmic contact with the p-doped GaN layer. A $30 \mathrm{~nm} / 100 \mathrm{~nm} \mathrm{Ti} / \mathrm{Au}$ bilayer was then sputter deposited to form the mirror/anode. This was followed by plasma-enhanced chemical vapor deposition (PECVD) of a 500 nm-thick $\mathrm{SiO}_{2}$ layer. The $\mathrm{SiO}_{2}$ layer was then patterned by reactive-ion etching (RIE) and the resulting hard mask was used to create mesa structures down to the n-GaN, using further RIE and inductively-coupled etching steps. This multi-step dry etching approach allows the formation of LEDs with selfaligned mirrors using only one photo-lithography step. The $\mathrm{SiO}_{2}$ hard mask was removed and a new $\mathrm{SiO}_{2}$ layer deposited by PECVD to serve as a passivation layer. It was then patterned to get access to the mesa structures. $\mathrm{Ti}(30 \mathrm{~nm}) / \mathrm{Al}(250 \mathrm{~nm}) / \mathrm{Ti}(30 \mathrm{~nm}) / \mathrm{Au}(100 \mathrm{~nm})$ layers were sputter-deposited and patterned by a standard lift-off process to connect the LEDs to wire bonding sites (fig. 1(b)). Laser dicing was used to form $100 \mu \mathrm{m}$ deep trenches around the probe. Each probe can be released by mechanically thinning (to $100 \mu \mathrm{m}$ ) from the back sapphire substrate and then bonded on a custom $\mathrm{PCB}$, the probe is wire bonded to allow each LED to be individually addressed. The whole device including PCB is electrically isolated by a $1 \mu \mathrm{m}$ thick transparent parylene layer, this layer is bio-compatible and protects the device from degradation. Due to the high pulse currents required for the LED good electrical shielding will be required to negate any electrical artefacts in neighboring recording probes.

Each LED was characterized electrically and optically. They can deliver a maximum of $1 \mathrm{~mW}$ of light, at an efficiency of $\sim 2 \%$. The average switch on voltage for the LEDs was $2.6 \pm 0.2 \mathrm{~V}(\mathrm{SD}, \mathrm{N}=23)$, while the average differential resistance was $680 \pm 0.2 \Omega(\mathrm{SD}, \mathrm{N}=23)$. Fig. 2 (a) shows the output power density - current relationship with (b) showing a typical spectrum from one of the LEDs. It has a peak emission at $446 \mathrm{~nm}$, shifting to 443 $\mathrm{nm}$ at higher current densities, with the full width half maximum increasing from $17 \mathrm{~nm}$ to $21 \mathrm{~nm}$. The emission spectrum overlaps well with the absorption spec- trum for ChR2. Finally the light intensity expected at various depths away from the probe was calculated using the model described in Bernstein et al [7] (fig. 2(c) and (d)). This model was supplemented with experimental data where the absorption through different thicknesses of brain slices was measured, the data was convolved with a Lambertian distribution to account for the sourcedetector distance remaining constant (fig. 2(d)). Brains were extracted from deeply-anaesthetised wild-type mice and sliced into coronal sections with a sliding microtome. All animal procedures were performed under license from the UK Home Office. Fig. 2(d) demonstrates the usefulness of this light source for both small and larger volume stimulation through irradiance modulation.
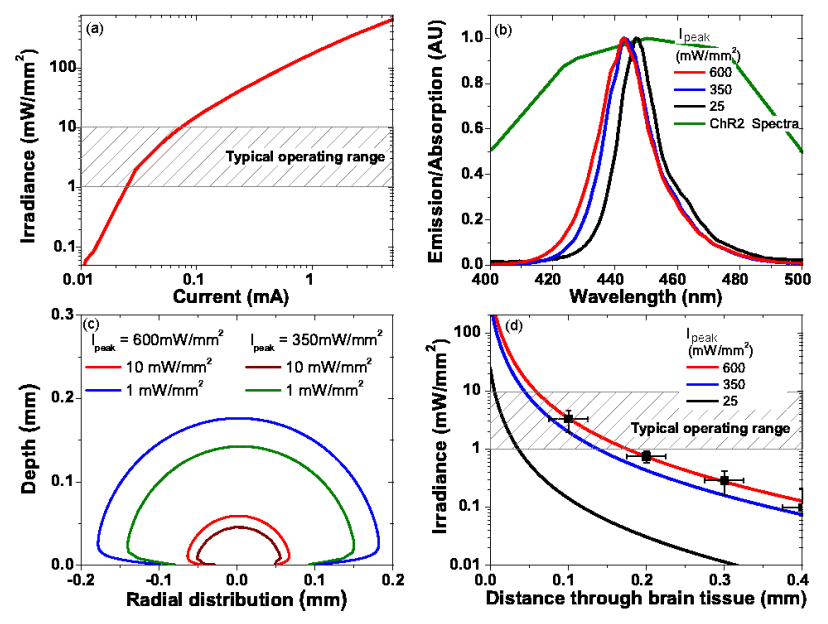

Fig. 2. (a) Irradiance-Current response for a LED. (b) Emission spectra of the LED at various light irradiances showing overlap with the absorption spectrum of ChR2. (c) \& (d) The calculated light intensity at differing depths within the brain for various device irradiances. The data points in (d) are from transmission experiments through varying thicknesses of brain slices (error is SD). Optogenetic constructs require $1-10 \mathrm{~mW} / \mathrm{mm}^{2}$ [1].

Temperature increases adversely affect the neuronal activity meaning that it is important to fully characterize the thermal properties of the device. Measurements were made using a thermal imaging camera (FLIR SC7000), with a close-up lens giving a spatial resolution of $\sim 5$ $\mu \mathrm{m}$. The camera outputs a voltage for each pixel and this voltage can be related to temperature through the materials emissivity. Due to the variety of materials used in the probe devices, some of which have poorly defined emissivity, a calibration measurement was performed. The device was allowed to reach thermal equilibrium at a series of temperatures and the voltage was recorded for each material at each temperature, this calibration allowed accurate measurement of the device temperature.

A conservative limit on the allowable temperature rise in brain tissue adjacent to the device was chosen to be $0.5^{\circ} \mathrm{C}$, based on the work of Andersen et al. who conclude that brain function should not be perturbed by a 
temperature rise of less than $1{ }^{\circ} \mathrm{C}[9]$. However, for a single pulse, higher temperature rises were manageable due to heat spreading through the device and not into the brain. By using finite-element analysis, the heat spreading from a LED during the on-phase was modeled. In particular the maximum rise of temperature recorded in brain tissue adjacent to the probe was estimated. The finite element model is approximating a solution to the heat equation; $\frac{\partial u}{\partial t}=\frac{k}{c_{p} \rho} \nabla^{2} u$. Here, $u$ is heat, $t$ is time, $k$ is thermal conductivity, $c_{p}$ is specific heat and $\rho$ is density. The specific heat and thermal conductivity of brain tissue were assumed to be $3650 \mathrm{~J} \mathrm{~kg}^{-1}{ }^{\circ} \mathrm{C}^{-1}$ and 0.5 $\mathrm{W} \mathrm{m} \mathrm{m}^{-1}{ }^{\circ} \mathrm{C}^{-1}$ respectively [10]. While the specific heat and thermal conductivity of the probe were assumed to be dominated by the relatively thick sapphire substrate, $756 \mathrm{~J} \mathrm{~kg}^{-1}{ }^{\circ} \mathrm{C}^{-1}$ and $23 \mathrm{~W} \mathrm{~m}^{-1}{ }^{\circ} \mathrm{C}^{-1}$ respectively.

Thermal modeling shows that heat is preferentially spread along the sapphire probe. This is due to the significantly higher thermal conductivity of sapphire compared to brain tissue. The large heat capacity of brain tissue also prevents its temperature rising significantly. From this modeling it was predicted that a $2{ }^{\circ} \mathrm{C}$ rise in temperature in the probe during a pulse, would lead to a $0.5{ }^{\circ} \mathrm{C}$ increase in the neighboring brain tissue.

The measured temperature rise on the surface of the LED for a single $200 \mathrm{~ms}$ pulse is shown in fig. 3(a) for two injection currents, $2 \mathrm{~mA}$ and $5 \mathrm{~mA}\left(350 \mathrm{~mW} / \mathrm{mm}^{2}\right.$ and $600 \mathrm{~mW} / \mathrm{mm}^{2}$ respectively). A single pulse, even at these durations, does not result in the $2{ }^{\circ} \mathrm{C}$ limit being exceeded. The maximum temperature from a single pulse of different durations is shown in fig. 3(b).

Optogenetic applications require multiple pulses and heat will accumulate in the LED under these conditions. Fig. 3(c) plots the average temperature rise at the surface of the LED (measured using the thermal camera) as a function of pulse repetition rate for multiple pulse widths driven at $5 \mathrm{~mA}$. Heat accumulates from the multiple pulses and the probe and brain will eventually reach thermal equilibrium, it is the average temperature that is important. This average temperature should not increase by more than $0.5{ }^{\circ} \mathrm{C}$. A similar experiment was also preformed to measure the temperature within a 400 $\mu \mathrm{m}$ brain slice using a thermocouple, the temperature rise measured were within experimental error of those in Fig. 3(c). An example operating scenario would be to require $10 \mathrm{~ms}$ pulses with an irradiance of $2 \mathrm{~mW} / \mathrm{mm}^{2}$ at around $100 \mu \mathrm{m}$ from the surface of the LED. This would require a current of $2 \mathrm{~mA}$ giving $350 \mathrm{~mW} / \mathrm{mm}^{2}$ at the LED surface (fig. 2 (d)) and places a $50 \mathrm{~Hz}$ limit on the pulse repetition rate (fig. 3 (d)). It should be noted that $1 \mathrm{~ms}$ pulse widths at $200 \mathrm{~Hz}$ is at the upper extremes for neuronal firing and $10 \mathrm{~ms}$ pulses at a repetition rate of $20 \mathrm{~Hz}$ is typical in optogenetic studies.

In conclusion, the optical and thermal characteristics of a GaN on sapphire LED probe have been explored and found appropriate for the field of optogenetics. This allows spatio-temporal activation of optogenetically targeted neurons with multiple optical sites in a compact

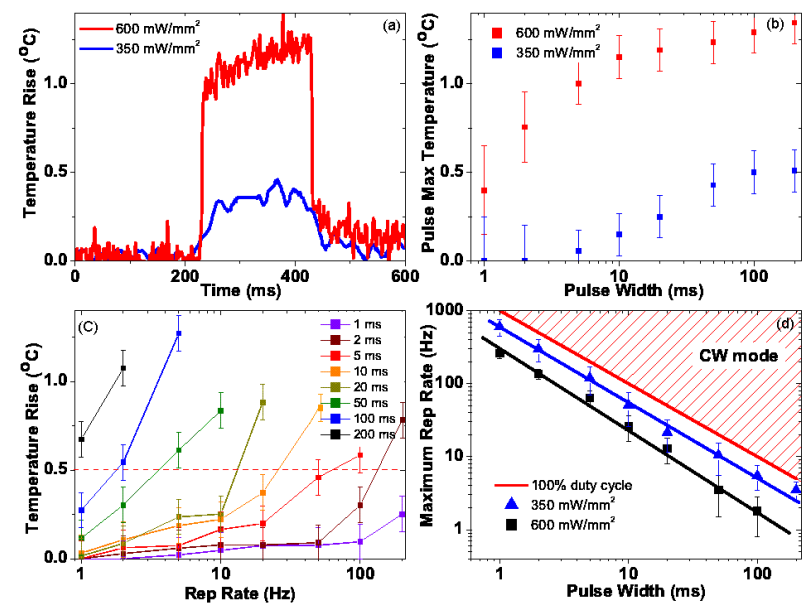

Fig. 3. (a) Measured temperature profile for a single 200 $\mathrm{ms}$ pulse at $350 \mathrm{~mW} / \mathrm{mm}^{2}$ and $600 \mathrm{~mW} / \mathrm{mm}^{2}$. (b) Measured peak temperature of a single pulse for various pulse widths. (c) Measured average temperature rise versus repetition rate for a LED operating at $600 \mathrm{~mW} / \mathrm{mm}^{2}$ with various pulse widths. (d) The maximum repetition rate that the device can be operated at and not rise $0.5{ }^{\circ} \mathrm{C}$ above ambient for two irradiances. Also shown is the maximum frequency for a given pulse. At lower irradiances the temperature rise was insufficient to be accurately recorded with the thermal camera.

integrated system without the need for expensive laser sources and complex optics.

The authors were supported by SU2P pilot project and EPSRC grants EP/D078555/1 and EP/F05999X/1.

\section{References}

1. K. Deisseroth, Nat Methods. 8, 26-9 (2011).

2. X. Liu, S. Ramirez, P. T. Pang, C. B. Puryear, A. Govindarajan, K. Deisseroth, and S. Tonegawa, Nature 484, 3815 (2012).

3. Y. Hayashi, Y. Tagawa, S. Yawata, S. Nakanishi, and K. Funabiki, Eur. J. Neurosci. 36, 2722-32 (2012).

4. E. Stark, T. Koos, and G. Buzsaki, J. Neurophysiol. 108 (2012).

5. A. N. Zorzos, J. Scholvin, E. S. Boyden, and C. G. Fonstad, Opt. Lett. 37, 4841-3 (2012).

6. M. Im, I.-J. Cho, F. Wu, K. Wise, and E. Yoon, in "IEEE EMBC," (2011), pp. $5480-3$.

7. J. G. Bernstein, X. Han, M. A. Henninger, E. Y. Ko, X. Qian, G. Talei Franzesi, J. P. McConnell, P. Stern, R. Desimone, and E. S. Boyden, in "Opt. Int. Tiss. \& Cells XIX," , vol. SPIE 6854 (2008), vol. SPIE 6854, pp. 68540-11H.

8. H. X. Zhang, D. Massoubre, J. McKendry, Z. Gong, B. Guilhabert, C. Griffin, E. Gu, P. E. Jessop, J. M. Girkin, and M. D. Dawson, Opt. Express 16, 9918-9926 (2008).

9. P. Andersen and E. I. Moser, Hippocampus 5, 491-8 (1995).

10. M. M. Elwassif, Q. Kong, M. Vazguez, and M. Bikson, J. Neural Eng. 3, 306-15 (2006). 